\title{
Exponential Mapping Function for Nonlinear Control*
}

\author{
Hildebrando de Castro*, Pedro Paglione, Carlos Ribeiro \\ Aeronautics Technological Institute, \\ Praça Marechal Eduardo Gomes, 50, São José dos Campos, 12.228-900, Brazil \\ hildebrando.castro@hotmail.com; paglione@ita.br; carlos@ita.br \\ * Corresponding author
}

\begin{abstract}
Industrial control engineers, who are often faced with the problem of dealing with projects involving unknown or poorly modelled systems, usually have at their disposal a limited number of options to develop, implement, and maintain controllers, namely PID and, lately, fuzzy-based controllers. The large use of the PID controller comes from the widespread knowledge of its theory and easy tuning methods and its prompt availability in control equipment and supervisory systems. Nevertheless, depending on the manufacturer's discretion, at least five gains have to be set. Fuzzy controllers for a SISO system usually demand between fifteen and twenty parameters. That's also a problem for the maintenance personnel. The proposed SISO controller needs two heuristically defined gains to be set, based on knowledge about the free response of the plant and expected disturbances. Its simplicity allows its implementation on devices with memory and processor constraints.
\end{abstract}

Keywords: Nonlinear control, Sliding-Mode Control, Fuzzy Logic Control.

\section{Introduction}

\section{Control Systems}

The fundamental control problem, according to [2] is " ... to find a technically feasible way to act on a given system or process so that it adheres, as closely as possible, to some desired behaviour. This approximate behaviour should be achieved in the face of uncertainty of the process and in the presence of uncontrollable external disturbances acting on the process" (italics belong to the authors). Worth noting here is that control should exhibit or approximate some behaviour without a perfect model of the process, defined by uncertainty in the amount and value of parameters and degree of modeling abstraction. A perfect model, furthermore, is practically impossible to obtain and, when a model is used, one should have in mind the constraints and trade-offs that are taken into consideration in its design.

This paper is then organized as follows:

- A brief discussion about issues with modelbased control;

- A way to streamline Sliding Mode Control (SMC);

- Prior works on how to dynamically tune an SMC;

\footnotetext{
* This paper is based on a previous presentation of the authors at the CBA 2012 (Brazilian Automation Congress) [1]. The simulation on this paper, however, incorporates all the necessary electrical signals conversions and incorporates enhancements in the controller developed thenceforth.
}

- A brief explanation of how a Fuzzy Logic Controller (FLC) can be simplified;

- The EMC (Exponential Mapping Function) derivation and its use in control;

- A procedure showing how to implement it in a real system;

- An example of its implementation on a rigorously simulated system;

- Conclusion.

\section{Issues with Model-based Control}

\section{Uncertainty on model dynamics}

The control project of a dynamical system can be based on its model or on different levels of prior knowledge about its free or closed-loop response. Model-based linear techniques for control abound, but systems and disturbances are usually of a nonlinear nature and can be extremely hard to model. Imprecisions may come from uncertainties about the plant and its external disturbances, or from a purposeful choice of model simplification [3], which may lead to poor or inadequate response in a modelbased control project.

Another advantage of analysing the response of a real, instrumented plant as input to the heuristic generation of initial gains or parameters to a controller is that conversions from digital to analogue signals, and viceversa, delays, and noises, are already being taken into account. Their modelling would be necessary in the building of an analytical 
model. Equipment and instrumentation aging, corrosion, deterioration etc., are unfortunately not taken into consideration when creating an analytical model. Usually these components are taken as ideal, or have their physical properties taken from the manufacturer's manual.

\section{Streamlining SMC}

EMC is partially based on ideas taken from SMC. Sliding Mode Control (SMC) is a technique to control nonlinear systems with uncertain dynamics and disturbances due to its order reduction property and low sensitivity to disturbances and process parameter variations, which relaxes the burden of the need for exact modeling. It does so by application of a discontinuous control signal that forces the system to slide along a specified surface of the systems normal behavior [4].

\section{Heuristics and simplification of an SMC}

From observations in [5] both $\lambda$ and $\phi$ can be derived using open loop experimental data. A switching band around the switching line can be used to alleviate chattering. Their idea is to apply maximal and minimal control input to a system and calculate $\lambda$ from the slope of the resulting curve. Figure 1 shows the open loop step response when applying maximal and minimal rudder angle on a boat.

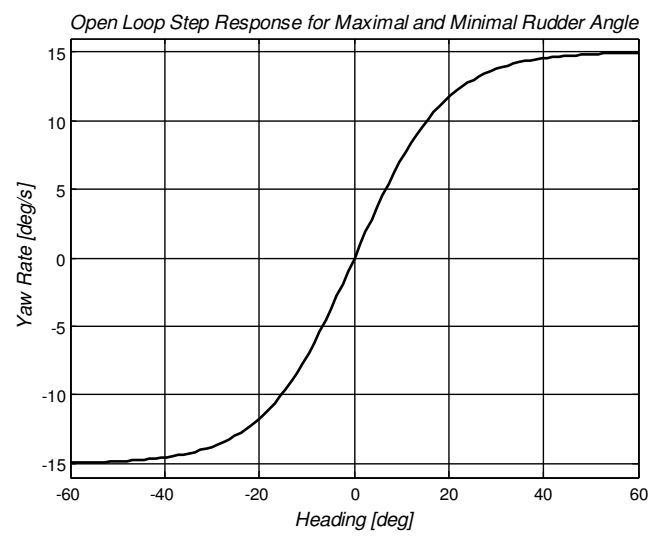

Figure 1. Open loop response to maximal and minimal rudder positions on a boat

Standard linear controller design can only linearly approximate this nonlinear curve. Additionally, the disturbance magnitude can be used to define $\phi$. This heuristic, or practical, method, allows for establishing initial values for the boundary layer of an SMC controller, thus eliminating the need of the equivalent control term and of an analytical model. The authors further discuss two problems associated with nonlinear time optimal controller design. First, how to get the true switching curve. For nonlinear systems, this switching curve is very difficult to get analytically. Secondly, how to approximate the nonlinear curve. To solve the first problem, they proposed to use system open loop experimental data. Under the maximal control command, the system output should be stabilized or saturated after a period of time. The nonlinear open loop response can be used as a switching curve since it represents the system's fastest response. To handle the second problem, an FLC can approximate the nonlinear switching curve since fuzzy systems are universal function approximators [6].

To further support this proposition, Pontryagin's maximum principle [7] states that for 2-dimensional time optimal controller design, there exists a nonlinear switching curve such that the control can have maximal value on one and minimal value on the other.

Following the idea of simplification, the authors in [8] proposed a simplified FLC (SFLC) based on the fact that most FLC implementations naturally inherit from conventional proportional-derivative (PD) or proportional-integral (PI) controllers. Observing that rule tables of most FLCs have skew-symmetric properties and that the absolute magnitude of the control input $|\mathrm{u}|$ or $\mid$ $\Delta \mathrm{u} \mid$ is proportional to the distance from its main diagonal line in the normalized input space, they derived a new variable called the signed distance:

$d_{s}=\frac{\dot{e}+\lambda \cdot e}{\sqrt{1+\lambda^{2}}}$

which is used as a sole fuzzy input variable. Here, a modified version of a sliding surface is being used instead of using $e$ and $\dot{e}$ as distinct entry parameters for a generic controller.

\section{Dynamic SMC Tuning}

The earliest applications of the time varying switching lines for the second order plant control are reported in [9] and [10]. The authors of these papers propose using switching lines which instantaneously change their position on the phase plane. The lines can move in one of the two ways: they may either rotate around the origin of the plane, or they may be shifted without changing their slope. This approach 
results in faster error convergence than the one obtained in conventional systems with fixed, time-invariant sliding lines.

The authors in [9] proposed a Moving Sliding Surface (MSS) that was dependent on the initial conditions of the system and through rotations and shifts would subsequently move it to a predetermined switching surface. In the proposed scheme, however, the sliding surface is shifted and/or rotated instantaneously, and after each movement the system representative point is no longer on the surface. Consequently, the system is, at least for some time, sensitive to disturbances and parameter uncertainties.

Another proposal [11] was to use a sigmoidal mapping function for a vision-based control of molten metal pouring.

The sigmoidal function with bias is:

$$
S(E-B)=\frac{1}{1+e^{-k \cdot(E-B)}}
$$

where $E$ is the error, $B$ is the bias, and $k$ is the response function shape parameter. The possible formats of the proposed sigmoidal function are shown with different gains, Figure $6 \mathrm{a}$, and bias, Figure $6 \mathrm{~b}, k \in[0.5,5]$ and $B \in[-10,10]$.
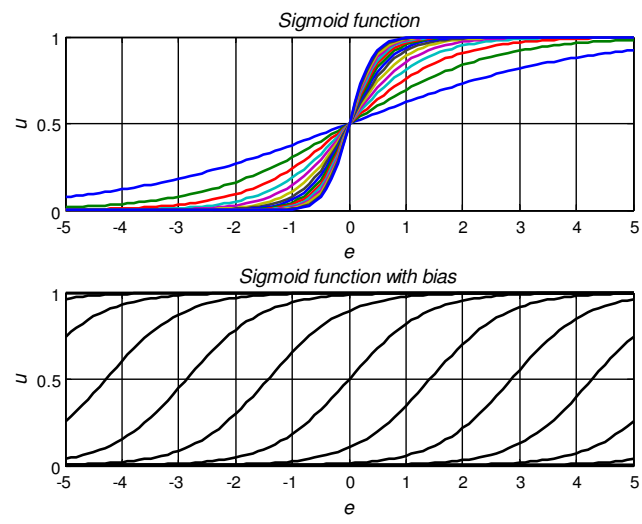

Figures 2a and 2b. Sigmoidal responses

The controller needs eleven parameters as inputs for the ten rules used for the adaptation of the controller gain $k$ and bias $B$. For the molten metal pouring system, adaptation was chosen to overcome the problems associated with the high nonlinearity of the process.

In [12] a way to tune a fuzzy SMC (FSMC) was derived by rotating and shifting the sliding surface and achieved faster reaching times and improved tracking error to a class of nonlinear systems compared with a conventional SMC.
The sliding surface can rotate or shift in the phase space in such a direction that the tracking behaviour can be improved. In their work it is shown that with arbitrary initial conditions, the reaching time and tracking error in the approaching phase can be significantly reduced. Chattering can also be reduced by tuning the fuzzy controller parameters.

Figure 3 presents the rotations and shiftings of the sliding line.

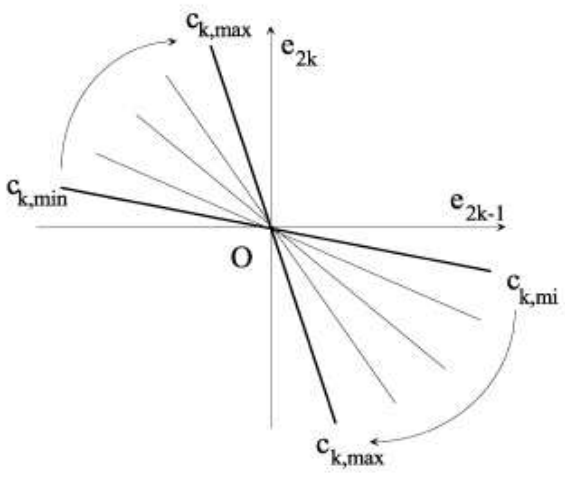

(a)

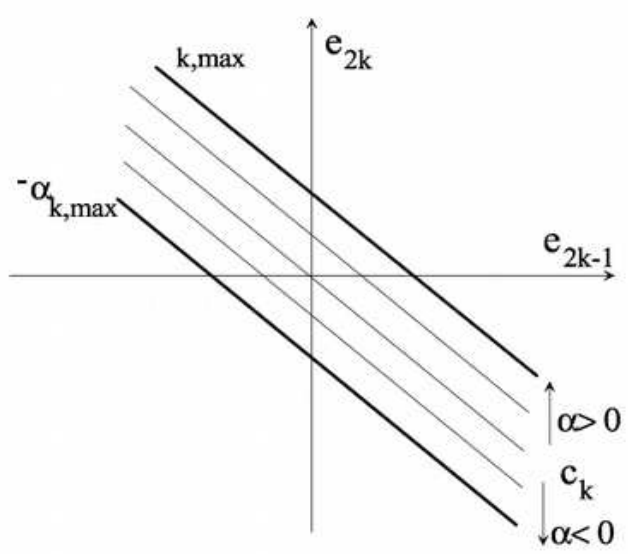

(b)

Figure 3a and 3b. Rotating and shifting the sliding surface to achieve faster response times

A drawback of this proposal is that the slope range of the rotating sliding surface and position range of the shifting sliding surface have to be known in advance. The stable zone of the phase plane is dealt with heuristics depending on the stiffness of the system to be controlled.

Another idea [13] was the use of a parabolic function coupled to a shifted sigmoid function to change the linear discontinuous control with the equivalent term computed with a simplified, model-based piecewise function. This nonlinear parabolic sliding surface was: 


$$
\hat{S}=s-k_{s}^{2}
$$

This function is illustrated in Figure 4. Here, $k_{s}$ defines the position of the parabolic surface. Any monotonous function can be selected to generate a $k_{s}$ value. For positive values of $k_{s}$, the nonlinear surface given in (3) is over the classical sliding line and for negative values it bends to the opposite side. The adaptive shifted sigmoid function was:

$$
k_{s}(t)=\frac{k_{s}^{+}-k_{s}^{-}}{1+e^{-m \cdot t+a}}+k_{s}^{-}
$$

Where $m$ is the time scaling parameter and $a$ is the time shifting parameter. The minimum and maximum allowable values of $k_{s}$ are $k_{s}^{-}$and $k_{s}^{+}$, respectively. This function computes the nonlinear sliding surface bending, which defines the controller performance. The parameters $k_{s}^{-}$and $k_{s}^{+}$are used to maintain the controller inputs inside an acceptable range but the authors didn't formalize how to compute or deduce them.

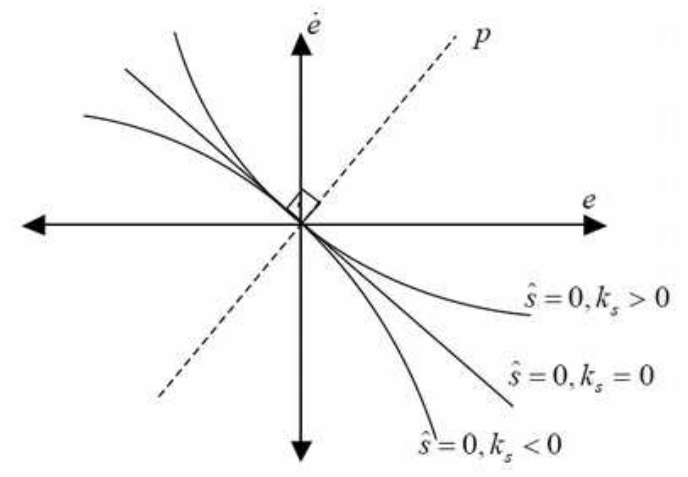

Figure 4. Nonlinear time-varying sliding surfaces in [11]

In [14] a parameterizable Modified Sigmoid Function (MSF) was proposed to nonlinearly interpolate in the boundary layer. Their motivation is that the sole use of a BL around the switching curve and then the use of a continuous control in its domain does not ensure the convergence of the state trajectory to the sliding surface and probably results in steady-state errors.

The MSF is:

$f(x)=-\frac{2}{1+e^{-\alpha x}}+1$

with $x \in\left[\begin{array}{ll}-1 & 1\end{array}\right]$ and $\alpha \in\left[\begin{array}{ll}0 & 10\end{array}\right]$. When $\alpha=0$ the response is a diagonal line.

The parameter $k$ that adapts the shape of the MSF is continuously tuned by five fuzzy rules of an FLC. The fuzzy inputs are the sliding surface $s$, and the current chattering $\Gamma=|\dot{s}|$. Its output is $\Delta \alpha$.

The fuzzy sliding control law is:

$u=\hat{u}+u_{f}$

with

$$
u_{f}=-k_{f u z z y}(e, \dot{e}, \lambda) \cdot \operatorname{sat}\left(\frac{s}{\phi}\right)
$$

with $k_{f u z z y}(e, \dot{e}, \lambda)$ as the absolute adaptive gain calculated by the FC and $\hat{u}$ is the nominal control law, dependent on the model of the plant.

In [15] the response within the boundary layer in an SMC was improved through dynamically deciding the slope of a linear sliding surface using fuzzy logic and sliding mode control $\left(\mathrm{FLSMC}_{\alpha}\right)$. The controller architecture to control a planar robot with two revolute joints is shown in Figure 5. $\theta$ and $\theta_{r}$ are the joint angle and reference angle, respectively.

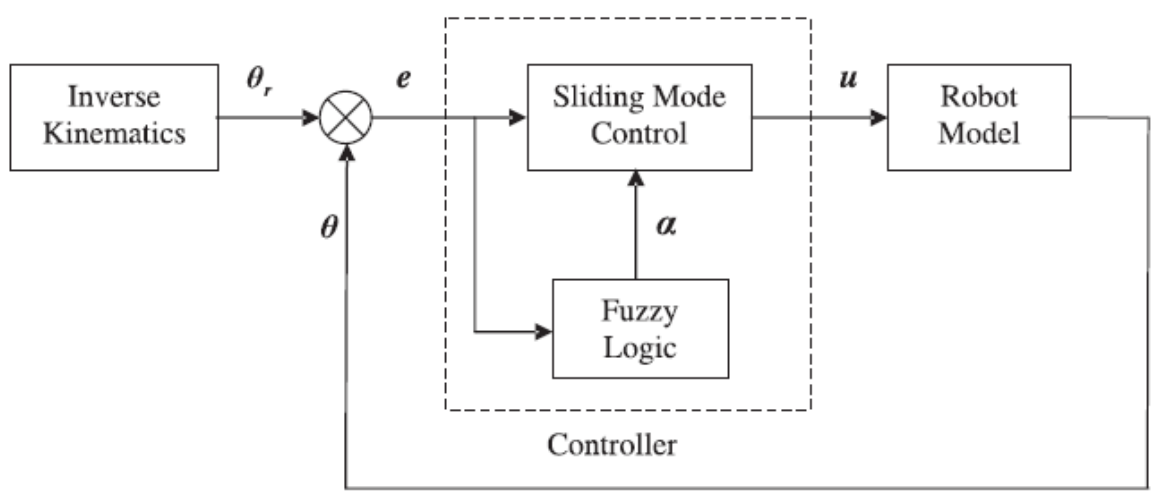

Figure 5. Controller architecture, showing the dependence on the inverse kinematics 
First, they developed a non-chattering standard robust sliding mode control using the inverse model of the plant. Then, in order to improve the performance of the controller a fuzzy logic algorithm was integrated with the sliding mode controller. The algorithm decides the slope of the sliding surface dynamically, as in Figure 6. Thus, the system is caught on the sliding surface rapidly and remains over it.

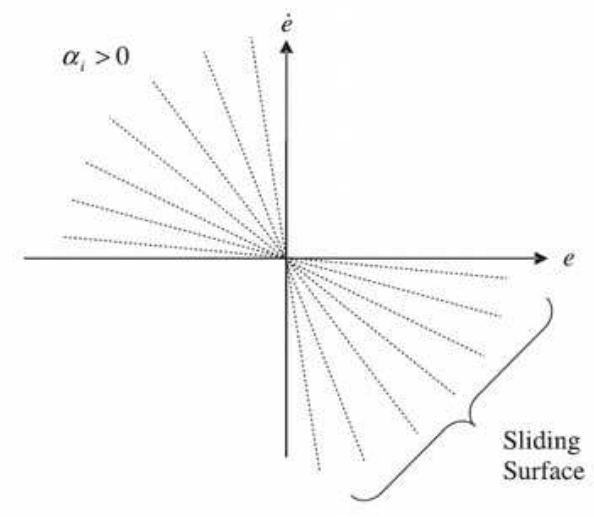

Figure 6. Sliding surface slope according to $\alpha$

In [16] a controller that uses a single-input $F L C$ with an one-dimensional rule base and seven linguistic fuzzy rules to continuously compute the slope of the sliding surface $\lambda$, through minimum and maximum values $\left[\begin{array}{ll}\lambda_{\min } & \lambda_{\max }\end{array}\right]$, was presented. This rotation is computed to decrease the reaching phase.

Figure 7 presents a graphical depiction of this movement.

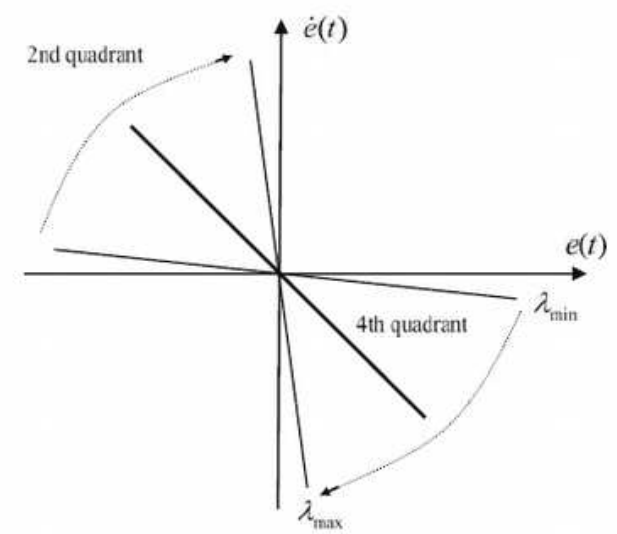

Figure 7. Sliding surface rotation governed by a single-input FLC

Lecture Notes [17] surveys theories and advances in the field of time-varying sliding modes. The reader is referred to this publication to further exploration of the subject.

Much importantly, all the approaches presented in Section 4, however, make use of the equivalent control and therefore need a model of the system.

\section{Fuzzy Logic Control}

Fuzzy logic, proposed by [18] in his seminal work for logic calculus and later extended in [19] is a way to approach problems that are difficult or unnatural to define in a binary, crisp sense.

\section{FLCs as an input-output mapping mechanism}

In [20] fuzzy controllers as an input-output mapping mechanism were discussed. The fuzzy rules are supposed to define the characteristic of a mapping $y=f(x)$. As fuzzy systems are universal approximators, systems are addressed which can approximate any mapping (function). This implies that:

$$
\forall x \in X,|F(x)-f(x)|<\varepsilon
$$

Where $F(x)$ is the function to be approximated and $\varepsilon$ can be chosen arbitrarily small. When we regard the fuzzy system as a discretization of $F(x)$, and know that between these discretizations interpolation is performed by means of fuzzy inference, it is clear that increasing the number of rules can provide a better approximation.

The fuzzy system can be regarded as an interpolation among a number of points, each defined by a fuzzy rule, under certain conditions. This mapping can be nonlinear as a result of the fuzzy rules, chosen operators, and membership functions (MFs).

\section{The EMC - Exponential Mapping Controller}

The number and format of the MFs, the rule base and the defuzzification method in an FLC allow for altering the shape of its response. This can be simplified by decreasing the input dimensionality and using only one shape-modifiable function. This in turn accounts for easier implementation since there is no need to define the amount and shape of the MFs as needed for an FLC and allows for faster computation times and more modest memory requirements, at the cost of a limited set of shapes and, consequently, a less expressive controller. 
EMC implements a bang-bang SISO SMCinspired approach with a heuristically-defined nonlinear mapping function equivalent to a bounded nonlinear sliding surface. Its boundary, $e_{r}$, and function shape, $u_{s}$, are derived from the open loop response of a stable plant, when maximal control inputs are applied. For dynamically stable or unstable systems, $u_{s}$ must be derived from previous knowledge about the plant behavior before instability happens.

The bang-bang method and the boundary layer solution serve as foundations in the creation of a novel controller that needs two heuristics that define the initial values of its two parameters. The first heuristic is to apply maximum control input to a stable plant and check the output for the steady state value and the time it took to reach it to define the error range parameter. This can be roughly compared to a saturation function inside a boundary layer. The second heuristic is to check the output shape, and then define the second parameter, the control input format, in a direct way. The idea here is to have the system return to zero error by following the same path it took to reach its limit, since this is its natural reaction.

\subsection{EMC Design}

Since in practice EMC needs only two parameters, its implementation and tuning are simplified. This simplicity allows for restoring the responsibility of tuning back to the systems operator or engineer. PIDs have three or more non-intuitive parameters to be tuned. Typical SISO FLCs, even though intuitive to implement, typically need at least nine parameters to be tuned. However, tuning only a small number of intuitive parameters is beneficial for the implementation, tuning and maintenance of automatic control systems in the industry. An alternative would be designing adaptable, untunable and hands-off controllers, but those depend on a high level of confidence - usually derived from design efforts with long duration and expensive costs - before its implementation can be carried out.

\subsection{EMC Implementation}

EMC is implemented as follows. First it calculates the error:

$e=y-y_{r}$

Then a switching line is computed:
$s_{E M C}=\frac{1}{e_{r}} \cdot e+\dot{e}$

where $e_{r} \neq 0$ is heuristically defined as being the acceptable error before applying full control input. It is comparable to the inverse of $\lambda$ in an SMC. For lower order or nonoscillatory systems the $\dot{e}$ term can usually be dropped. Then $s_{E M C}$ is finally restricted to $-1 \leq s_{E M C} \leq 1$ to be within the exponential function allowable range:

$s_{E M C}=\left\{\begin{array}{cl}-1, & \text { if } s_{E M C}<-1 \\ s_{E M C}, & \text { if }-1 \geq s_{E M C} \leq 1 \\ 1, & \text { if } s_{E M C}>1\end{array}\right.$

And then an exponential function is computed:

$u_{e}=\operatorname{sign}\left(s_{E M C}\right) \cdot\left(\left(1-\left|s_{E M C}\right|\right)^{\frac{1}{2}}-1\right)$

where

$-10 \leq u_{s} \leq 10$

is the parameter that alters the exponential function from a concave to a convex shape, allowing for a class of nonlinearities and disturbances to be dealt with.

Finally, the controller input is calculated:

$u_{E M C}=\frac{u_{\max }-u_{\min }}{2} \cdot\left(u_{e}-1\right)+u_{\max }$

where $u_{\min }$ and $u_{\max }$, collectively called $u_{r}$, for controller input range, are the actuator minimum and maximum values, respectively.

$u_{r}=\left[\begin{array}{l}u_{\min } \\ u_{\max }\end{array}\right]$

Figure 8 presents the sliding lines $s_{E M C}$ and controller outputs $u_{E M C}$ or $\Delta u_{E M C}$ for the EMC, with $e_{r}=1$, shapes $-4 \leq u_{s} \leq+4, u_{\min }=-1$, $u_{\max }=1$, and $-1 \leq s_{E M C} \leq 1$.

For systems that need a strong control effort even with small errors, $u_{s}$ should be defined in the range $0 \leq u_{s} \leq 10$, or $-10 \leq u_{s} \leq 0$ otherwise.

This modified exponential function in its basic form allows for the controller to have strong actuation for small errors. This is related to the sigmoidal functions proposed in [11], [13], and [14], but the exponential mapping is more flexible in the sense that it can also generate a weak actuation for higher errors. 


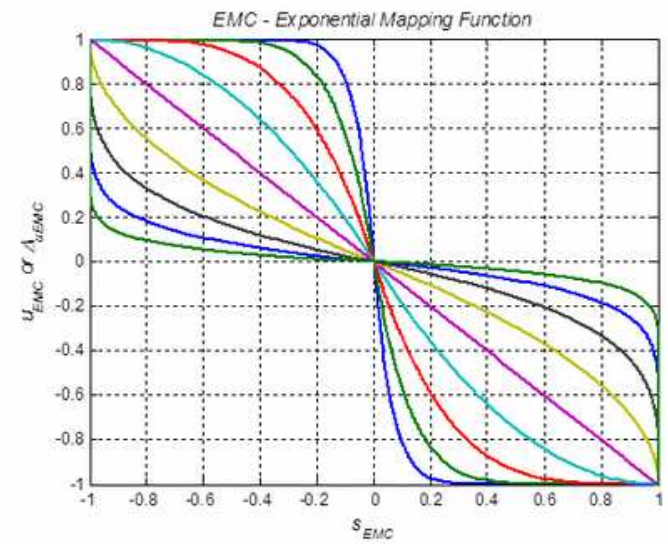

Figure 8. Plot of $s_{E M C}$ and $u_{E M C}$, $u_{s} \in\{-4,-3, \ldots, 0, \ldots,+4\}$

\subsection{Handling asymmetries in actuators}

Membership functions for an asymmetrical valve are not reflexed, since valves close faster than they open. The exponential function as defined in (12) cannot mimic this behavior, because it is perfectly mirrored about the horizontal and vertical axes in Cartesian quadrants I and III. This asymmetry can be seen in Figure 9, where symmetrical MFs are compared to original, asymmetrical, valve responses.

To solve this issue, the following changes are proposed to EMC.

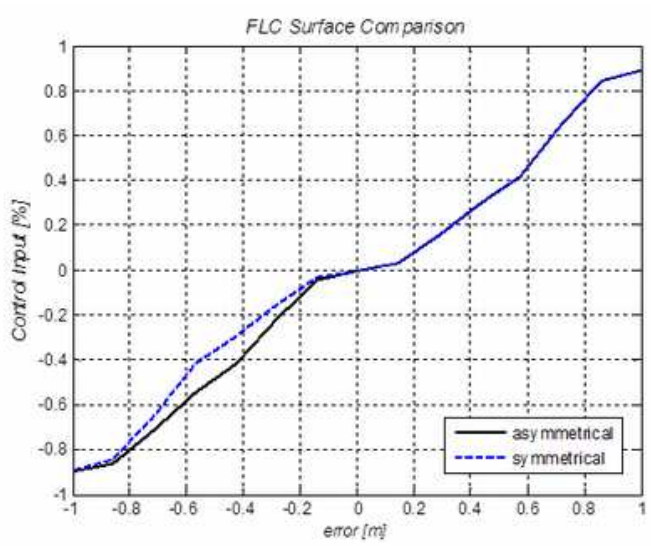

Figure 9. Comparison between symmetrical and asymmetrical MFs

Derive a new variable:

$u_{\text {as }}=\left[u_{\text {ast }} u_{\text {asu }}\right]$

where $u_{a s t}$ defines the negative, or lower, control input shape and $u_{a s u}$ defines the positive, or upper, control input shape. For this new configuration, a new test is needed:

$u_{s}=\left\{\begin{array}{l}u_{a s l}, \text { if } s_{E M C} \leq 0 \\ u_{a s u}, \text { if } s_{E M C}>0\end{array}\right.$
Figure 10 presents an example of the asymmetrical EMC, with $-2 \leq u_{a s l} \leq 6$ and $-4 \leq u_{a s t} \leq 4, e_{r}=1, u_{\min }=-1, u_{\max }=1$, and $-1 \leq s_{E M C} \leq 1$.

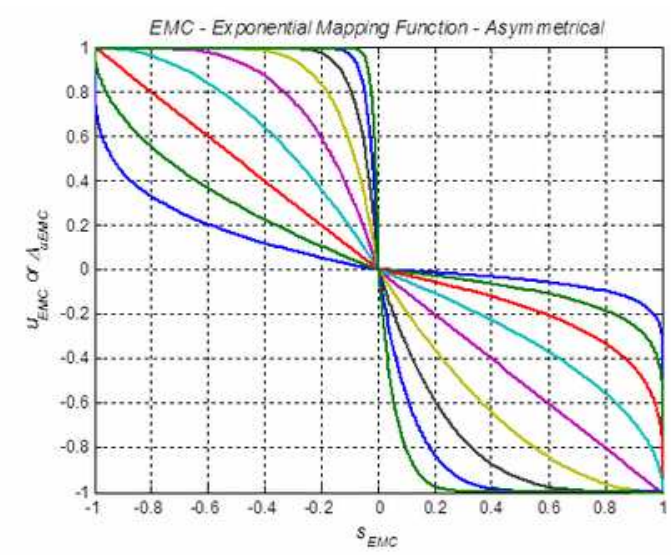

Figure 10. Asymmetrical EMC Response

For the Tank Level Control [22], the asymmetrical EMC was implemented using $u_{a s l}=2.4$ and $u_{a s u}=1.7$. The comparison in responses can be seen in Figure 11. The valve is now opening faster, as shown by the overshoot in the upper reference. There is no change in the lower reference, since it does not depend on the opening of the valve, but on its closing. The remaining parameters are $e_{r}=1 \mathrm{~m}$ , $u_{\min }=-1$, and $u_{\max }=1$.
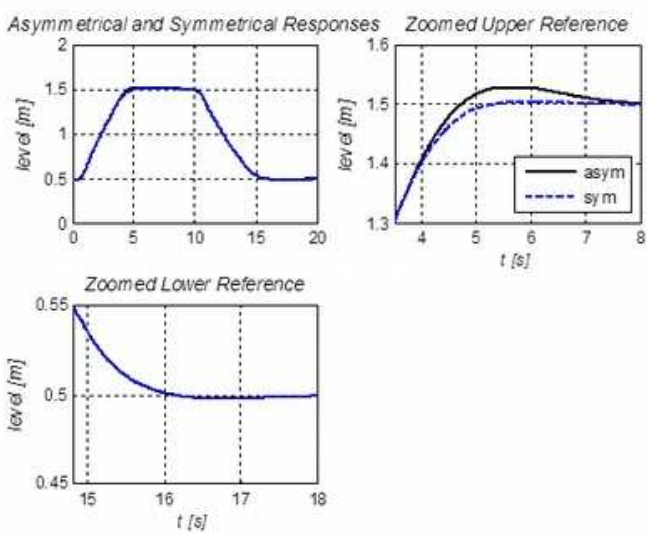

Figure 11. Asymmetrical Tank Level EMC Response

Since this is an infrequent situation, the author decided to use equation (14) for the implemented system here.

\subsection{Positional and incremental forms of EMC}

EMC is able to perform the positional an incremental forms for control, meaning that it can produce $u$ or $\Delta u$, as its output. 
Equation (14) is for the positional implementation. For the incremental form equation (14) becomes equation (18):

$$
\begin{aligned}
u_{E M C_{k+1}} & =u_{E M C_{k}}+ \\
& +\left(\frac{\Delta u_{\max }-\Delta u_{\min }}{2} \cdot\left(u_{e}-1\right)+\Delta u_{\max }\right) \cdot T
\end{aligned}
$$

where $u_{E M C_{k}}$ and $u_{E M C_{k+1}}$ are the controller inputs in discrete time $k$ and $k+1$, respectively and $\Delta u_{\min }$ and $\Delta u_{\max }$ are the actuator minimal and maximal changes during the sampling period $T$. Collectively,

$$
\Delta u_{r}=\left[\begin{array}{l}
\Delta u_{\min } \\
\Delta u_{\max }
\end{array}\right]
$$

and

$$
\Delta u_{r}=u_{r} \cdot T
$$

Strictly speaking, $u_{r}$ or $\Delta u_{r}$ could be thought as parameters for the EMC but since $e_{r}$ and $u_{s}$ dominate the tuning process, the author decided to leave them as values that can be defined directly from the manufacturer's or from the system specification, in a cascade controller, for example.

The sampling period, and consequently the control input period, depends on the closed-loop bandwidth of the system [20]. $T$ is dependent on the system and actuator dynamics or in practice as a restriction in the control hardware, and therefore will not be considered as another controller parameter. At best it can be considered as a choice, almost an option, for the controller designer, based on its experience with the plant and hardware at hand. DCSs, for example, have a few sampling rates available, perhaps three, to be able to couple with all the different systems and processes that will be controlled.

\subsection{EMC procedure}

Below is the procedure that is used to specify an EMC controller to a SISO system.

Step 1. Define $\operatorname{sgn}\left(e_{r}\right)=\operatorname{sgn}\left(\frac{\Delta y}{\Delta u}\right)$;

Step 2. Choose if the control is positional or incremental;

Step 3. If the control is incremental, define the control period $T$;

Step 4. Define the control inputs $u_{\min }$ and $u_{\max }$ or $\Delta u_{\min }$ and $\Delta u_{\max }$ according to the manufacturer's specification (usually expressed in volts or amperes, or volts/cycle, amperes/cycle) or the system control input limits themselves, if it is a cascade controller, for example;

Step 5. Apply minimal and maximal control input in open loop. Wait for the system to reach its steady state for both cases (or right before saturation, if the system is unstable) and calculate $e_{r}=\frac{\Delta y}{\Delta t}$;

Step 6. For $u_{s}$, visually check the open loop response and use a corresponding value. Check for asymmetries in the minimal and maximal responses and, if needed, apply asymmetric control inputs;

Step 7. Run the EMC controller in closed loop with the new $e_{r}$ and $u_{s}$ and observe the system response;

Step 8. Go to step 6 and change $e_{r}$ and $u_{s}$

\begin{tabular}{|c|c|c|}
\hline Choice & Response & Action \\
\hline Decrease $e_{r}$ & Strong & $\begin{array}{l}\text { Full control input } \\
\text { applied earlier }\end{array}$ \\
\hline Increase $e_{r}$ & Weak & $\begin{array}{l}\text { Full control input } \\
\text { applied earlier }\end{array}$ \\
\hline Decrease $u_{s}$ & Smooth & Steadily stronger \\
\hline Increase $u_{s}$ & Crispy & Immediately strong \\
\hline
\end{tabular}
according to Table 1 until a satisfactory result is achieved.

Table 1. Behavior of EMC Parameters

\section{Practical Implementation with EMC}

A two-tank system, based on the Coupled Tanks experiment from Quanser®, in [23], was chosen as a platform to test EMC in a simulated environment with electrical noise in sensors and actuators, quantization effects, filtering, external disturbances, and changes in the model, in an effort to bring real-world difficulties and constraints to the proposed controller. The simulation was written in MATLAB $\AA$, using its scripting facilities. The goal is to control the level of tank 2 through the water pump, using tank 1 in cascade mode. Figure 12 shows a simplified schematics of the device. 


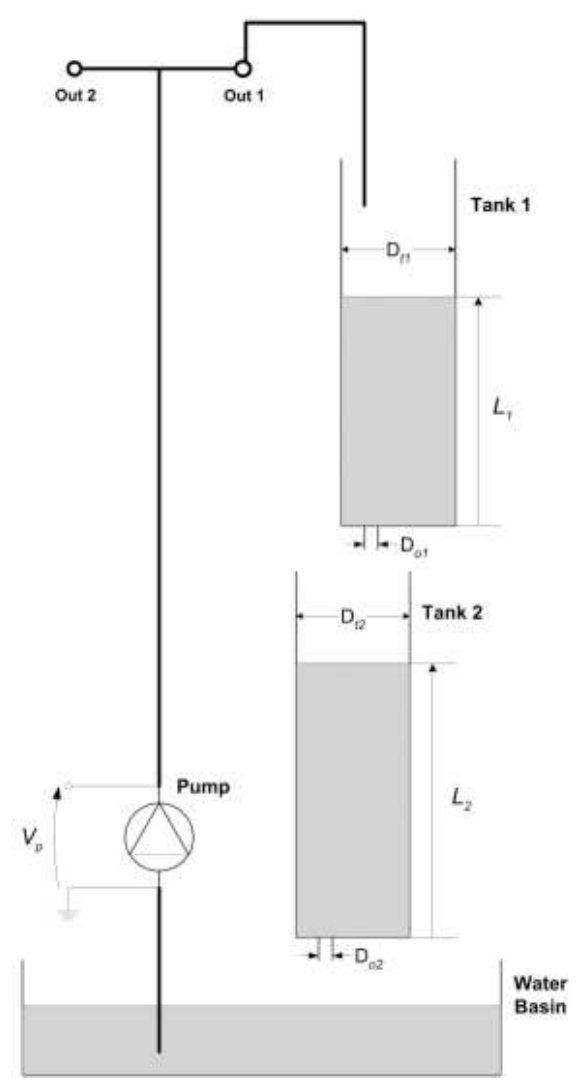

Figure 12. Coupled Tanks Experiment from Quanser ${ }^{\circledR}$

A detailed system, since its first voltage reading from tank 2 water level pressure sensor, until its control input voltage generation to the DC motor in the water pump was simulated. Gains, saturations, delays, quantization, filtering, noise from sensors, noise from actuator, and external disturbances were incorporated in the model. Input actuator noise, input sensor noise, plant noise, and output sensor noise are the four possible noises in a control system [24], two of which are being taken into account, the input actuator noise and the output sensor noise. There are no other measured inputs but the controlled one and the plant noise, like bubbles, water flow friction etc., are not being modeled.

The system consists of two tanks with orifices and level sensors at the bottom of each tank, a pump, and a water basin. The two tanks have the same diameters and can be fitted with different outflow orifices. For this simulation, the medium orifices were used.

A disturbance tap allows the operator to pour water from tank 1 directly into the basin.

The dynamic equations for the liquid level in the two tanks are in derived as in [22].

\section{Closed Loop Process}

The control process starts with the reading of a noisy pressure sensor voltage at the bottom of tank 2 every $T=0.01 \mathrm{~s}$. Level of tank 1 is not being used. Electrical noise is represented by white noise with zero mean and variance $\sigma^{2}=0.01$ [21]. The noisy voltage is quantized by a 12-bit ADC and then converted to a digital representation.

Quantization is an unwanted effect cause by the finite precision of a digital converter, who has a specific amount of bits available to convert an analogue signal to a digital train of bits [24]. Quantization of a signal inserts noise, or error, in a control system. Filtering is one of the available resources to counteract this problem.

The quantization equation used is simple and straightforward [23]:

$$
V_{q}(k)=q_{L} \cdot \text { round }\left(\frac{V_{n}}{q_{L}}\right)
$$

where $q_{L}$ is the quantum size, the result of the following equation:

$$
q_{L}=\frac{V_{p_{\text {max }}}-V_{p_{\text {min }}}}{2^{A D C_{r}}}
$$

where $V_{\max }$ is the maximal voltage from the sensor, $V_{\min }$ is its minimum, and $A D C_{r}$ is the resolution of the ADC.

Quantization generates noise, and filtering is necessary. Quanser proposes, for the pressure sensor, a continuous filter with a $0.33 \mathrm{~Hz}$ cut-off frequency. With the filtered voltage, a conversion to centimetres eases the design of the controller, and this value will be used as input to the controller, to further calculation of the error.

The reference used as input to $E M C_{c}$, the one used for control, is calculated with the aid of EMC itself, named $E M C_{\text {ref }}$, using error:

$e(k)=L_{2_{n f}}(k)-L_{2_{f}}(k)$

EMC can be used as a reference generator because it is a function mapping. The parameters for $E M C_{c}$ are $e_{r}=12 \mathrm{~cm}$, $u_{r}=[-0.0050 .005]$, and $u_{s}=1$.

The derivative of the error is calculated in $E M C_{\text {ref }}$ by:

$\dot{e}(k)=\frac{e(k)-e(k-1)}{h}$ 
It was expected that this calculation would cause noise in the system, but the smoothing of the level sensor noise was enough to keep the system stable so no further filtering was necessary.

The output of $E M C_{\text {ref }}$ is the input to $E M C_{c}$, without the need of the error derivative. $E M C_{c}$ parameters were $e_{r}=5 \mathrm{~cm}$, $u_{r}=\left[\begin{array}{ll}-20 & 20\end{array}\right] \cdot T$, and $u_{s}=5$.

$E M C_{c}$ is the control input, so this real value has to be sent to the water pump DC motor via a DAC. $E M C_{c}$ already computes its control input in the actuator voltage range, so no specific gain was necessary. A DAC with a 12bit resolution was used to generate a quantized value. Then, a saturation to impose limits on the voltage output is required, between $-20 \mathrm{~V}$ and $20 \mathrm{~V}$. After saturation, the signal is again noisy, because of the quantization, so we have to smooth it out, using a discrete filter with a $3 \mathrm{~Hz}$ cut-off frequency, proposed by QUANSER. To simulate most of the aspects of the real system, noise was then added to the filtered voltage, with a PSD of 0.001 .

The results of the simulated system for setpoint changes are presented in Figure 13. The initial conditions are $L_{1}(0)=9 \mathrm{~cm}$, $L_{2}(0)=9 \mathrm{~cm}$, and $V_{m}(0)=5.14 \mathrm{~V}$.

The disturbance tap is pressed from $150 s<t<152 s$, a two second removal of water from tank 1 to the basin, and the orifice diameter is changed from the medium to the large size when $t>700 s$, a $16.67 \%$ change in the model definition.
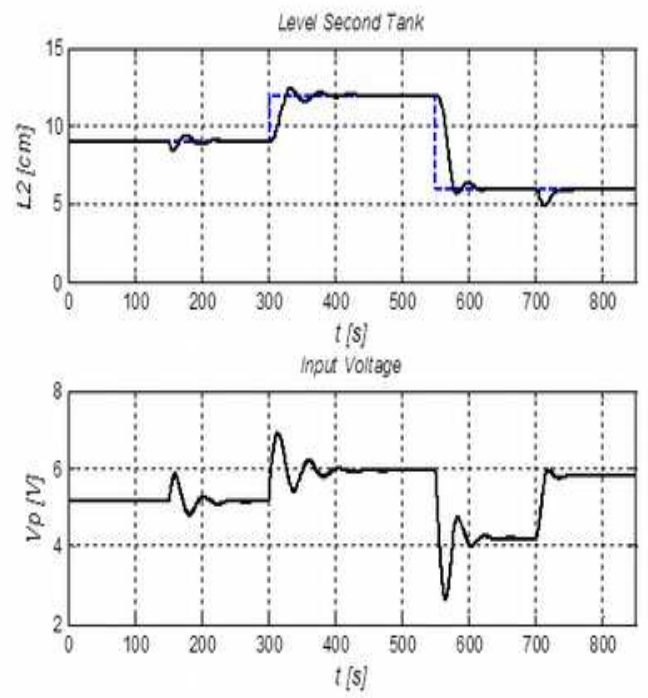

Figure 13. Two-tank response to set-point changes
The results of the simulated system for a sinusoidal signal tracking is presented in Figure 14. The applied disturbance and model change are the same as the previous run. The reference sinusoidal is:

$$
L_{2_{r f}}(k)=-4 \cdot \sin \left(\frac{k}{120}\right)+9
$$
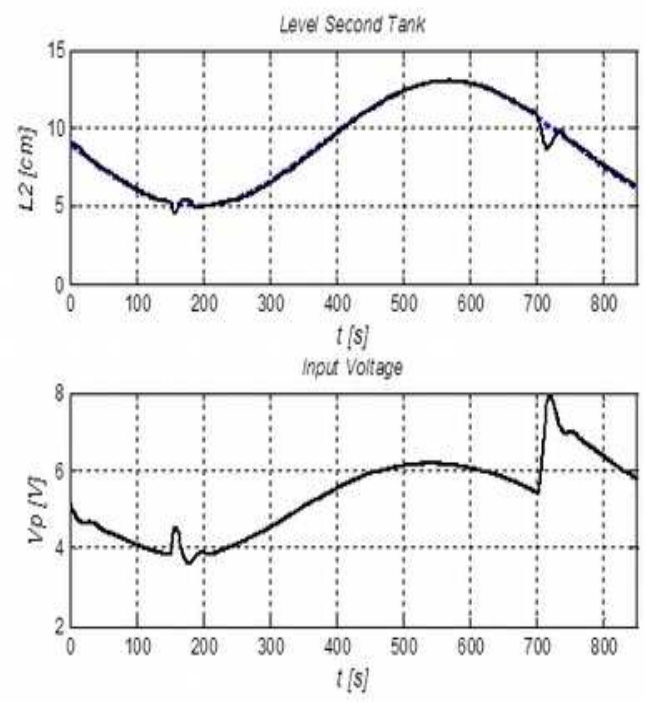

Figure 14. Two-tank response to a sinusoidal reference

It should be noted that a simple low-pass filter of cut-off frequency $2.5 \mathrm{~Hz}$ is added to the output signal of the tank 2 level pressure sensor. This filter is necessary to attenuate the high-frequency noise content of the level measurement. Such a measurement noise is mostly created by the sensor's environment consisting of turbulent flow and circulating air bubbles.

\section{Conclusion}

An exponential-function based controller inspired by the bang-bang SMC and FLC was developed. It seeks to ease the task of implementing and maintaining a nonlinear SISO control system. EMC exhibits the property of allowing its tuning using only two parameters. The first, $e_{r}$, is the most important one, since during the simulations it was seen that its value can be critical in terms of stability, but a heuristic helps estimating its initial values. The second parameter, $u_{s}$, allows for specifying a smoother or a more aggressive response. Some of the controllers showed good results with the very first estimate of their parameters, obtained from simulation. EMC has also been implemented in a laboratory real system, namely the Quanser 3DOF Helicopter. 
The controller did not rely on any analytical information about the simulated systems but only on their open loop response and behavior. Using a Lyapunov approach to check and validate its stability properties and robustness is intended for further development of the proposed controller.

\section{REFERENCES}

1. CAStro Filho, H. F., P. Paglione, C. H. C. Ribeiro, Exponential Mapping Function for Control, XIX Congresso Brasileiro de Automática, Campina Grande, Paraíba, Brazil, 2012.

2. GOODWIN, G. C.; S. F. GRAEBE, M. E. SALGADO, Control System Design, Upper Saddle River, New Jersey, Prentice Hall, 2001.

3. Slotine, J. J., W. LI, Applied Nonlinear Control. New Jersey, Prentice Hall, 1991.

4. UTKIN, V. I., Sliding Regimes and Their Applications in Variable Structure Systems. Moscow, 1978, MIR Publ.

5. SONG, F., S. M. SMITH, Design of Sliding Mode Fuzzy Controllers for an Autonomous Underwater Vehicle without System Model. OCEAN 2000 MTS/IEEE Conf. and Exhibition, 2000.

6. KOSKO, B., Fuzzy Systems as Universal Approximators. IEEE Transactions on Computers, vol. 43(11), Nov. 1994.

7. IYANAGA, S., Y. KAWADA, Pontrjagin's [sic] Maximum Principle. Encyclopedic Dictionary of Mathematics. Cambridge, MA: MIT Press, §88C, 1980, pp. 295-296.

8. CHOI, B.-J., S.-W. KWAK, B. K. KIM, Design and Stability Analysis of SingleInput Fuzzy Logic Controller. IEEE Transactions on Systems, Man, and Cybernetics 2000 - Part B, Cybernetics. vol. 30, no 2., pp 303-309.

9. YAMAMOTO, S., I. HASHIMOTO, Present Status And Future Needs: The View From Japanese Industry. Chemical Process Controls CPC IV: Proceedings of the Fourth International Conference on Chemical Process Control, AIChE: New York: 1991. Arkun, Y., Ray, W. H., Eds.

10. CHOI, S. B., D. W. PARK, Moving Sliding Surfaces for Fast Tracking Control of
Second-order Dynamical systems. ASME Journal of Dynamic Systems, Measurement, and Control, vol. 116, 1994, pp. 154-158.

11. TABATABAEI, E., A. GUEZ, H. CHOI, Adaptive Sigmoidal Molten Metal Pouring Control. IEEE Transactions on Control Systems Technology, 1998, Vol. 6, No. 2.

12. HA, Q. P., D. C. RYE, H. F. DURRANTWHYTE, Fuzzy Moving Sliding Mode Control with Application to Robotic Manipulators. Automatica, vol. 35, 1999, pp. 607-616.

13. TOKAT, S., I. EKSIN, M. GUZELKAYA, Sliding Mode Control Using A Nonlinear Time-Varying Sliding Surface. Lisbon, Portugal: 2002. Proceedings of the 10th Mediterranean Conference on Control and Automation - MED2002.

14. KIM, Y.-K., G. J. JEON, Error Reduction of Sliding Mode Control Using SigmoidType Nonlinear Interpolation in the Boundary Layer. International Journal of Control, Automation, and Systems, 2004. Vol. 2, no. 4, pp 523-520.

15. YAGIZ, N., Y. HACIOGLU, Fuzzy Sliding Modes with Moving Surface for the Robust Control of a Planar Robot. Journal of Vibration and Control, 2005. Vol. 11, No. 7, pp. 903-922.

16. YORGANCIOĞLU, F. H. KÖMÜRCÜGIL, Single-input Fuzzy-like Moving Sliding Surface Approach to the Sliding Mode Control. Electrical Engineering, 2008, vol. 90(1-2), pp. 199-207.

17. BARTOSZEWICS, A. A. NOWACKALEVERTON, Time-Varying Sliding Modes for Second and Third Order Systems. Lecture Notes in Control and Information Sciences, Springer-Verlag, Heidelberg, 2009.

18. ZADEH, L., Fuzzy Sets. Information and Control, vol. 8, 1965, pp. 338-353.

19. ZADEH, L., Fuzzy Logic and Approximate Reasoning. Synthese, vol. 30, 1975, pp. 407-428.

20. JAGER, R. Fuzzy Logic in Control. PhD Thesis Technische Universiteit Delft. Netherlands, 1995. 
21. FRANKLIN, G. F., J. D. POWELL, A. EMAMI-NAEINI, Feedback Control of Dynamic Systems. Seventh Edition. Chapter 8. Prentice-Hall, 2014.

22. MATHWORKS, Inc. Control of a TwoTank System. Robust Control Toolbox. Available on http://www.mathworks.com/ help/robust/examples/control-of-a-two-tanksystem.html. Accessed on Dec. 14, 2014.
23. QUANSER, Inc. Quanser_Coupled Tanks_System_Specificatons_Generic.pdf. Available on http://www.quanser.com/ Products/coupled_tanks. Accessed on November, 25th, 2014.

24. ÅSTRÖM, K. J., B. WITTENMARK, Computer-Controlled Systems: Theory and Design. $3^{\text {rd }}$ Ed., Dover Books, 2013. 